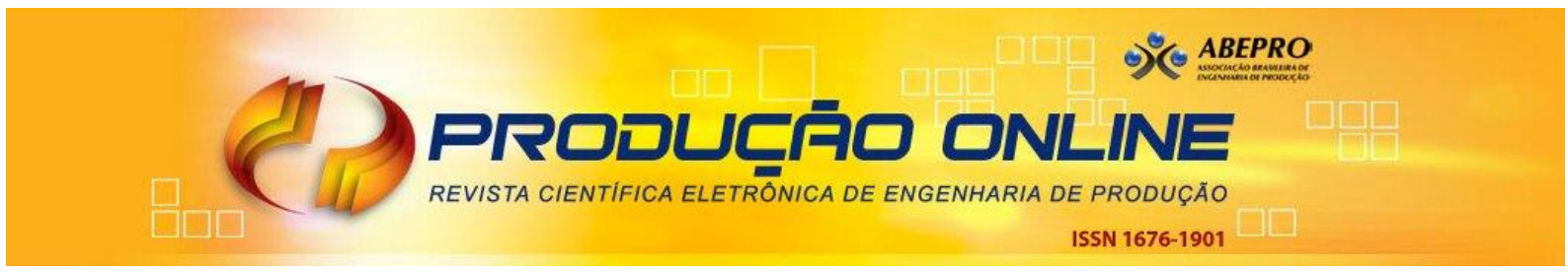

\title{
BENEFÍCIOS E BARREIRAS PARA ACEITAÇÃO DE MEDIDORES INTELIGENTES RESIDENCIAIS
}

\section{BENEFITS AND BARRIERS FOR HOUSEHOLD SMART METERS' ACCEPTANCE}

\author{
Jonathan Gumz*E-mail: jonathan.gumz@gmail.com \\ Diego Castro Fettermann* E-mail: d.fettermann@ufsc.br \\ *Universidade Federal de Santa Catarina (UFSC), Florianópolis, SC, Brasil
}

Resumo: Programas de implantação de medidores inteligentes nas residências são fundamentais para se atingirem as metas de modernização da rede elétrica e uso racional das fontes de energia. Porém, além da implantação dos aparelhos nas residências é necessária a aceitação dos consumidores, que possuem papel fundamental na redução do uso de energia elétrica. $O$ objetivo deste estudo é investigar na literatura quais fatores influenciam positivamente (benefícios) e negativamente (barreiras) para a aceitação. Para isso, foi realizada uma revisão sistemática da literatura usando o método PRISMA (Preferred Research Items for Systematic Review and MetaAnalyses). Foram analisados 172 artigos, dos quais 42 são estudos aplicados. Os resultados sugerem 26 fatores diferentes que influenciam a aceitação, sendo os benefícios mais importantes "melhor gerenciamento de energia através do feedback", "eco-preocupação" e "expectativa de ganho financeiro", já as barreiras mais relevantes são "segurança ameaçada", "falta de familiaridade" e "custos associados". As pesquisas de aceitação estão ainda muito concentradas na Europa, EUA e Austrália, sendo necessário que países em desenvolvimento, que lideram a expansão do uso de fontes renováveis em suas matrizes energéticas, também considerem investigar a aceitação local de medidores para obter sucesso em suas implementações.

Palavras-chave: Medidores inteligentes. Aceitação. Método PRISMA.

Abstract: The implementation of smart meters in homes is essential to achieve the goals of modernization of the electricity grid and rational use of energy sources. However, in addition to the implantation of devices in homes, it is necessary to have the consumers' acceptance due to their fundamental role in reducing electricity usage. This study investigates in the literature which factors influence it positively (benefits) and negatively (barriers) for acceptance. For this, a systematic literature review was carried out using the PRISMA method (Preferred research items for systematic review and meta-analyses). A total of 172 articles were surveyed, of which 42 are applied studies. The results obtained 26 different factors that influence acceptance, with the most important benefits being "better energy management through feedback," "eco-concern," and "expectation of financial gain." In contrast, the most relevant barriers are "threatened security," "lack of familiarity," and "associated costs." Acceptance surveys are still highly concentrated in Europe, the USA, and Australia. It is necessary that developing countries, which are leading the expansion of renewable sources in their energy matrices, also consider investigating the local acceptance of meters to be successful in their implementations.

Keywords: Smart meters. Acceptance. PRISMA Method. 


\section{INTRODUÇÃO}

O sistema elétrico vigente foi desenvolvido para atender a um consumo constante, uma produção centralizada e cujo pico de produção esteja alinhado com o de consumo (ELLABBAN; ABU-RUB, 2016). Estas características não atendem aos requisitos atuais, que apresentam uma parcela crescente da energia produzida por fontes renováveis, que apresentam uma produção intermitente e dependente de condições meteorológicas (ELLABBAN; ABU-RUB, 2016). Além disso, se verifica uma necessidade de comunicação de retroalimentação bidirecional em tempo real, por meio de fibra óptica e redes wireless, informando ao mesmo tempo o quanto é gerado pelas fontes fósseis, renováveis, consumidores, além de informar o consumo (ELLABBAN; ABU-RUB, 2016). Para este cenário é preciso o desenvolvimento de uma nova rede elétrica capaz de fazer uso eficiente das fontes de energia renováveis (HOSSAIN et al., 2016), também denominada de rede inteligente (smart grid).

No contexto de implementação da rede elétrica inteligente, o medidor residencial de energia elétrica consiste em um importante equipamento desse sistema. O medidor inteligente de energia é resultado de uma evolução na infraestrutura para medição da eletricidade consumida (AVANCINI et al., 2019). Enquanto o medidor eletromecânico possui as funções de leitura e cobrança manual, conexão física e mostrador analógico, o medidor inteligente passou a incluir funções de cobrança automatizada de tarifa, armazenamento e gerenciamento de dados, comunicação entre cliente e fornecedor, detecção e diagnóstico de falhas no sistema, tela interativa (IHD) e conexão sem fio (AVANCINI et al., 2019). Neste contexto, diversas iniciativas de implementação em larga de medidores inteligentes estão sendo realizadas na Austrália (BEAL et al., 2018), Taiwan, Indonésia, Coréia do Sul e Vietnã (CHOU et al., 2015), Alemanha (ARNOLD et al., 2018), Finlândia (PAlHO et al., 2018), Reino Unido (BUCHANAN et al., 2016) Canadá, Nova Zelândia, Austrália, Estados Unidos (BUCHANAN; RUSSO; ANDERSON, 2015), Arábia Saudita (DÜŞTEGÖR et al., 2018) entre outros.

Durante a última década foram realizadas diversas pesquisas para investigar os fatores que influenciam a aceitação de medidores inteligentes pelos 
consumidores (e.g. KRISHNAMURTI et al., 2012; CHOU et al., 2015; BUCHANAN et al., 2016; BUGDEN; STEDMAN, 2019). Apesar destas iniciativas, diversos projetos de implementação de medidores inteligentes ainda seguem com a suposição de total aceitação dos medidores inteligentes pelos consumidores, mesmo que esta suposição seja amplamente rejeitada na literatura (e.g., BERTOLDO; POUMADÈRE; RODRIGUES, 2015; BUCHANAN et al., 2016; THRONDSEN, 2017; BUGDEN; STEDMAN, 2019; KUMAR, 2019). A literatura indica a necessidade de compreender melhor os fatores que afetam a aceitação dos consumidores em relação às tecnologias da rede inteligente, como no caso do medidor inteligente (ELLABBAN; ABU-RUB, 2016). O processo de implementação dos medidores inteligentes deve considerar ativamente 0 consumidor para 0 atingimento dos objetivos de implementação (YANG et al., 2019), sendo que a sua implementação sem a devida consideração do consumidor pode gerar grupos de resistência (HESS; COLEY, 2014; JEGEN; PHILION, 2017) e atrasos nos projetos de implementação (GUERREIRO et al., 2015). Entretanto, a literatura registra uma notável ausência de estudos de aceitação de medidores inteligentes no Brasil e na América do Sul.

Em razão da importância consumidor para o funcionamento pleno da rede inteligente, este artigo tem como objetivo apresentar os fatores que contribuem na intenção comportamental de uso de medidores inteligentes residenciais. Para tanto, foi realizado um levantamento na literatura sobre casos de implementação de medidores inteligentes, além dos benefícios e barreiras identificados na implementação dos medidores inteligentes residenciais. Por fim, este artigo busca apresentar subsídios para o melhor planejamento dos projetos de implementação de medidores inteligentes, reunindo e discutindo os principais fatores que determinam a efetividade de sua implementação em unidades residenciais.

\section{DESENVOLVIMENTO}

\subsection{Método}

A revisão sistemática de literatura é um método de coleta de dados empíricos. Entre os métodos para revisão sistemática, se destaca o PRISMA. Neste 
trabalho foram utilizados como base os procedimentos recomendados no PRISMA e aplicados em outros estudos na área de engenharia (MORENO-BLANCO et al., 2019; AYODELE; CHANG-RICHARDS; GONZÁLEZ, 2020; GAO et al., 2020), que são divididos em cinco etapas (Figura 1). A seguir são detalhados os procedimentos realizados em cada uma das cinco etapas do método.

Figura 1 - Tópicos de Método para Busca PRISMA

\begin{tabular}{l|l} 
1. Protocolo de \\
Palavras-chave
\end{tabular}$\quad \begin{gathered}\text { 2. Critérios } \\
\text { de } \\
\text { Elegibilidade }\end{gathered} \quad \begin{aligned} & \text { 3. Fontes de } \\
& \text { Informação }\end{aligned}>$ 4. Busca $\quad>\begin{gathered}5 . \text { Seleção } \\
\text { dos Estudos }\end{gathered}$

Para a revisão sistemática foram estabelecidos dois campos de busca, 0 primeiro relacionado à medidores inteligentes e o segundo à sua aceitação por parte dos clientes. Ao final da etapa exploratória foram definidas as seguintes palavraschave na busca sistemática de literatura: "smart meter", "intelligent meter" referentes ao medidor inteligente e "acceptance", "acceptation", "engagement" e "adoption" relacionadas à sua aceitação por parte dos clientes. Esta busca foi realizada considerando título, resumo e palavras-chave nos artigos (topic).

Foram utilizadas como fontes de informação as bases de dados Scopus, Science Direct e Web of Science, como é de frequente em pesquisas da área (e.g., ALKAWSI; ALI; ALGHUSHAMI, 2018; MORENO-BLANCO et al., 2019; AYODELE; CHANG-RICHARDS; GONZÁLEZ, 2020; GAO et al., 2020). Os resultados apresentados se referem a busca realizada na data de 11 de março de 2020. Foram selecionados estudos na forma de artigos em inglês publicados em periódicos. Após a remoção das duplicidades, foram obtidos 184 estudos diferentes, sendo que 10 destes não apresentaram foco na aceitação de medidores inteligentes residenciais e dois não foram localizados, resultando no número final de 172 artigos analisados na íntegra (Figura 2).

A partir da análise dos artigos selecionados são apresentados fatores que, segundo esta literatura, podem influenciar a aceitação de medidores inteligentes por parte dos consumidores. Estes fatores foram separados em dois grupos: benefícios e barreiras São denominados de benefícios os fatores que aumentam a chance de aceitação dos medidores inteligentes, enquanto as barreiras são os fatores que 
diminuem a aceitação. Cada um dos fatores ainda foram organizados de acordo com as dimensões de aceitação de novas tecnologias de energia (BOUDET, 2019): (I) tecnologia, (ii) pessoas, (iii) lugar e (iv) processo.

Figura 2 - Fluxo de informações protocolo PRISMA

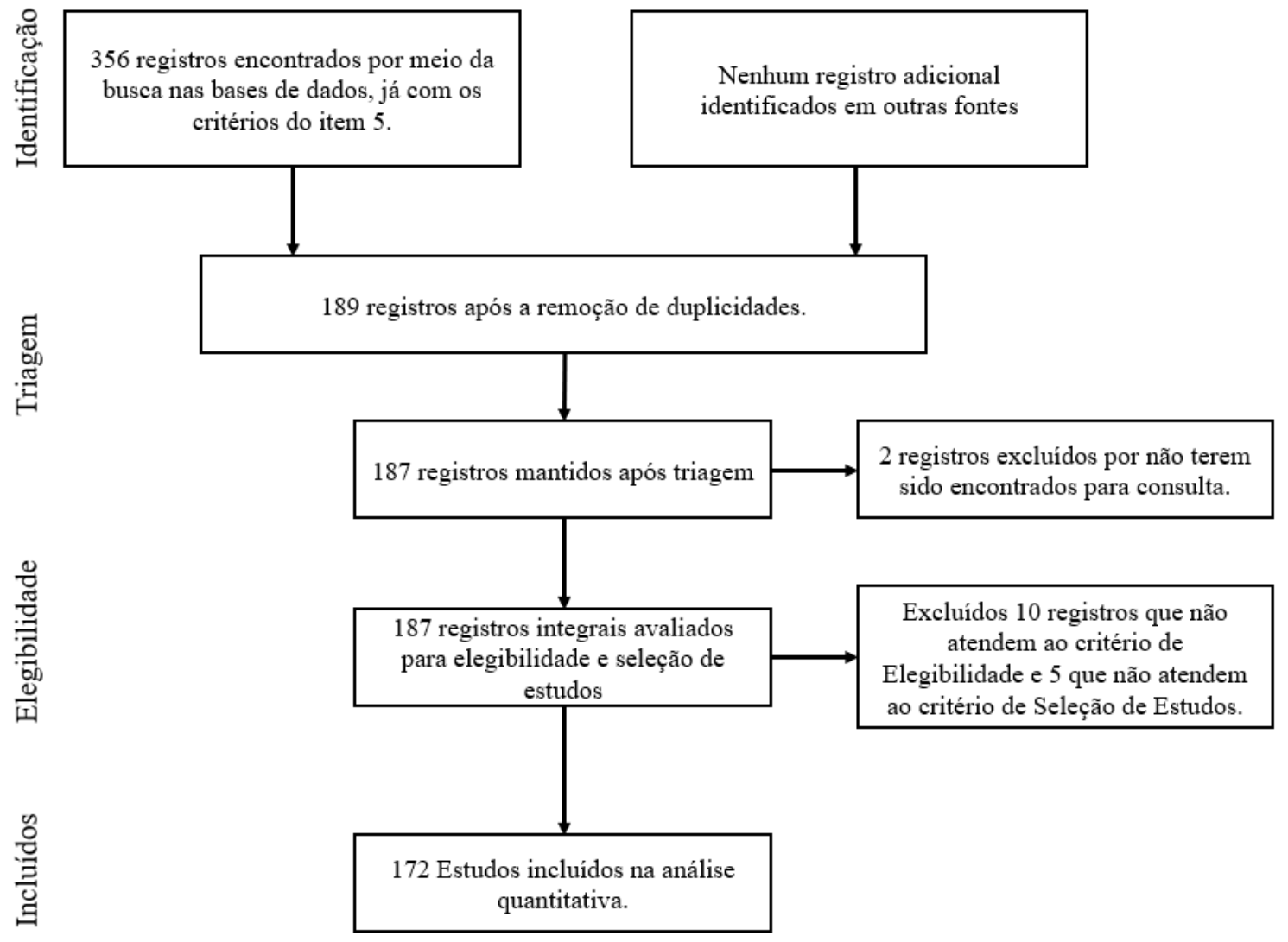

\subsection{Resultados}

\subsubsection{Mapeamento de pesquisas aplicadas}

O levantamento identificou um total de 42 estudos publicados focados na compreensão na aceitação dos medidores inteligentes em uma determinada região. A Figura 3 apresenta a localização geográfica de cada um destes estudos realizados. As pesquisas realizadas nestas cidades incluem aplicação de questionários, realização de entrevistas, criação de grupos focais, estudos de caso e experimentos sociais e de campo. Se verifica uma maior concentração de estudos 
na Europa, Estados Unidos, Austrália e sudeste Asiático. A Europa apresenta uma maior concentração de estudos em razão da diretriz para implementação de medidores inteligentes em $70 \%$ das residências dos países do bloco europeu (EUROPEAN UNION, 2009). Na Austrália se concentram os estudos para a implementação de medidores inteligentes para o controle do consumo de água (e.g., BEAL; FLYNN, 2015). A compreensão dos fatores importantes para a aceitação de medidores inteligentes em países em desenvolvimento ainda é pouco explorada, com exceção de estudos realizados na África do Sul (BOOYSEN; VISSER; BURGER, 2019) e Indonésia (CHOU; YUTAMI, 2014).

Figura 3 - Pesquisas aplicadas de aceitação de medidores inteligentes

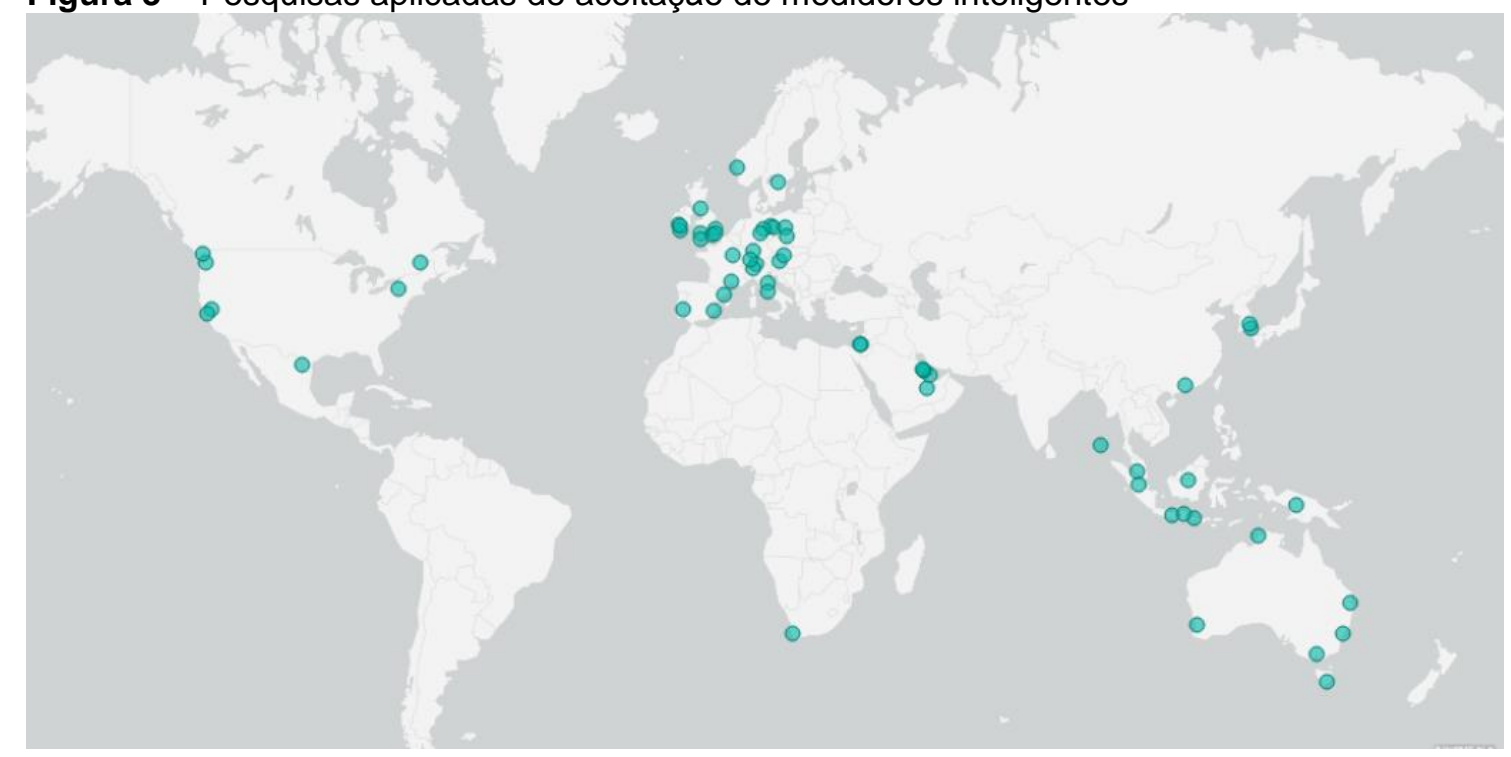

2.2.2 Benefícios para aceitação de medidores inteligentes

Foram identificadas 369 citações de fatores considerados como benefícios associados a implementação de medidores inteligentes (Tabela 1) e (

Tabela 2). Entre estes a dimensão pessoas é a mais frequente, seguida pela de tecnologia, processos e lugar. 
Tabela 1 - Fatores considerados como benefícios para aceitação de medidores inteligentes

Fator

Freq.

(DARBY, 2010; OLMOS et al., 2011; KAUFMANN; KÜNZEL; LOOCK, 2013; BOYLE et a1., 2013; CHOU; YUTAMI, 2014; DAVIES et a1., 2014; DYSON et a1., 2014; HESS; COLEY, 2014; PARK; KIM; KIM, 2014; RIXEN; WEIGAND, 2014; BALTA-OZKAN; AMERIGHI; BOTELER, 2014; BEGIER, 2014; CHOU et al., 2015; GUERREIRO et al., 2015; MATSCHOSS;
KAHMA; HEISKANEN, 2015; BALLO, 2015; CAMPILO et al., 2016; BUCHANAN et a1., 2016; CHEN; XU; ARPAN, 2017. GARRAB; BOUALLEGUE; BOUALLEGUE, 2017; MARCH

Utilidade

percebid

매응 Energia limpa (Carbon Saving)

Facilidade de uso percebida

Integração com outras tecnologias

Abandono da Energia Nuclear

Melhor

do uso de

energia atraves

总
37

et al., 2017; MOGLES et al., 2017; WARKENTIN; GOEL; MENARD, 2017; WARREN, 2017; WILSON; HARGREAVES; HAUXWELL-BALDWIN, 2017; ZHOU; BROWN, 2017;

ALKAWSI; ALI, 2018; ALKAWSI; ALI; ALGHUSHAMI, 2018; ALSKAIF et al., 2018; GRANDCLEMENT; NADAİ, 2018; MONTGINOUL; VESTIER, 2018; SCHOPFER; TIEFENBECK
STAAKE, 2018; BARBOUR; GONZÁLEZ, 2018; CHAWLA; KOWALSKA-PYZALSKA; WIDAYAT, 2019; BOUDET, 2019; CHAWLA; KOWALSKA-PYZALSKA; ORALHAN, 2020; VAN DE KAA et al., 2020)

(OLMOS et a1., 2011; MURTAGH et al., 2013; HESS, 2014; JARAMILLO; CARDONA; HENAO, 2014; MARQUES; BENTO; COSTA, 2014; BALTA-OZKAN; AMERIGHI; BOTELER, 20 2014; DAVIES et al., 2014; DEGEN; MOSTASHARI; MAURER, 2014; THRONDSEN; RYGHAUG, 2015; BURYK et al., 2015; ESPLUGA et al., 2016; MOGLES et al, 2017; ALKAWSI; ALI; ALGHUSHAMI, 2018; ALSKAIF et al., 2018; HIEL SCHER; SOVACOOL, 2018; NOVAK et al., 2018;
BUGDEN; STEDMAN, 2019)

(MEYERS; WILLIAMS; MATTHEWS, 2010; FENSEL et al., 2013; PARK; KIM; KIM, 2014; RIXEN; WEIGAND, 2014; CHOU; YUTAMI, 2014; DAVIES et a1., 2014; FENSEL; KUMAR;

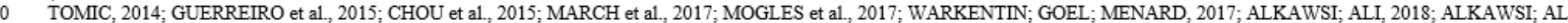
ALGHUSHAMI, 2018; MONTGINOUL; VESTIER, 2018; ALSKAIF et a1., 2018; DÜŞTEGÖR et a1., 2018; SAREEN; ROMMETVEIT, 2019; BOUDET, 2019; VAN DE KAA et al., 2020) (DARBY, 2010; SUNIKKA-BLANK et al., 2012; HESS, 2014; HESS; COLEY, 2014; PARK; KIM; KIM, 2014; MATSCHOSS; KAHMA; HEISKANEN, 2015; VASSILEVA; CAMPILLO, 2016; ALKAWSI; ALI; ALGHUSHAMI, 2018; SIL VAST et al., 2018; BOUDET, 2019; CHAWLA; KOWALSKA-PYZALSKA; WIDAYAT, 2019; VAN DE KAA et al., 2020; CHAWLA; KOWALSKA-PYZALSKA; ORALHAN, 2020; CHAWLA; KOWALSKA-PYZALSKA; SIL VEIRA, 2020)

(CHEN; YEH, 2017)

(HARTWAY; PRICE; WOO, 1999; DARBY, 2010; MEYERS; WILLIAMS; MATTHEWS, 2010; OLMOS et al., 2011; GIACOMIN; BERTOLA, 2012; KRISHNAMURTI et al., 2012 SUNIKKA-BLANK et al., 2012; GERPOTT; PAUKERT, 2013; KAUFMANN; KÜNZEL; LOOCK, 2013; MURTAGH et a1., 2013; OL TRA et al., 2013; STEWART et al., 2013; CHOU; YUTAMI, 2014; DAVIES et al., 2014; DYSON et al., 2014; FENSEL; KUMAR; TOMIC, 2014; BAL TA-OZKAN; AMERIGHI; BOTELER, 2014; JARAMILLO; CARDONA; HENAO, 2014; BEGIER, 2014; TORRITI, 2014; CHOU et al., 2015; BALLO, 2015; GUERREIRO et al., 2015; MATSCHOSS; KAHMA; HEISKANEN, 2015; NACHREINER et al., 2015; BUCHANAN; 62 RUSSO; ANDERSON, 2015; CAMPILLO et al, 2016; HALL; JEANNERET; RAI, 2016; RAIMI; CARRICO, 2016; VASSIL EVA; CAMPILLO, 2016; WOLISZ et al., 2016; BUCHANAN et SCHWEDE, 2017; WARKENTIN; GOEL; MENARD, 2017; WILSON; HARGREAVES; HAUXWELL-BALDWIN, 2017; ZHOU; BROWN, 2017; ABDMOULEH; GASTLI; BENBRAHIM, 2018; ALSKAIF et al., 2018; HIELSCHER; SOVACOOL, 2018; NOVAK et a1., 2018; PAIHO et a1., 2018; PETERS; AXSEN; MALLETT, 2018; SIL VAST et a1., 2018; CHABÉFERRET et al., 2019; CHAWLA; KOWALSKA-PYZALSKA, 2019; CHAWLA; KOWALSKA-PYZALSKA; WIDAYAT, 2019; KOWALSKA-PYZALSKA; BYRKA, 2019; SAREEN;
ROMMETVEIT, 2019; YANG et al., 2019; BOUDET, 2019; BROWN; MARKUSSON, 2019; CAHN; KATZ; GHERMANDI, 2020; CHAWLA; KOWALSKA-PYZALSKA; ORALHAN, 2020; CHAWLA; KOWALSKA-PYZALSKA; SIL VEIRA, 2020; VAN DE KAA et al., 2020)

(MEYERS; WILLIAMS; MATTHEWS, 2010; OLMOS et a1., 2011; BOYLE et al., 2013; FENSEL et al., 2013; GERPOTT; PAUKERT, 2013; KAUFMANN; KÜNZEL; LOOCK, 2013; MURTAGH et al., 2013; RIXEN; WEIGAND, 2013; CHEN; DELMAS; KAISER, 2014; DAVIES et al., 2014; HESS; COLEY, 2014; BAL TA-OZKAN; AMERIGHI; BOTELER, 2014; PARK; KIM; KIM, 2014; BEGIER, 2014; BALLO, 2015; MATSCHOSS; KAHMA; HEISKANEN, 2015; NACHREINER et al., 2015; THRONDSEN; RYGHAUG, 2015; BUCHANAN et al. 2016; ESPLUGA et al., 2016; MICHAELS; PARAG, 2016; RAIMI; CARRICO, 2016; BEAL; GURUNG; STEWART, 2016; SøNDERLUND et al., 2016; VASSILEVA; CAMPILLO, 2016; CHEN; XU; ARPAN, 2017; MOGLES et a1, 2017. MOSER 2017. WARKENO BOAIT et al., 2017; ALK AWSI; ALI; ALG Get SOVACOOL, 2018; LIU; MUKHEIBIR, 2018; NOVAK et al., 2018; PAIHO et al., 2018; PETERS; AXSEN; MALLETT, 2018; RAUSSER; STRIELKOWSKI; ŠTREIMIKIENE, 2018; RYGHAUG; SKJøLSVOLD; HEDENREICH, 2018; SILVAST et al., 2018; WEMYSS et al., 2018; BIGERNA et al., 2018; BUGDEN; STEDMAN, 2019; CHABE-FERRET et a1., 2019; CHAWLA; KOWALSKA-PYZALSKA, 2019; CHAWLA; KOWALSKA-PYZALSKA; WIDAYAT, 2019; GUO; LAM; LI, 2019; KOWALSKA-PYZALSKA; BYRKA, 2019; SAREEN; ROMMETVEIT, 2019; BOUDET, 2019; CAHN; KATZ; GHERMANDI, 2020; CHAWLA; KOWALSKA-PYZALSKA; ORALHAN, 2020; VAN DE KAA et al., 2020)

Revista Produção Online. Florianópolis, SC, v. 21, n. 1, p. 131-156, 2021 
Tabela 2 - Fatores considerados como benefícios para aceitação de medidores inteligentes

(conclusão)

(FARUQUI; HARRIS; HLEDIK, 2010; OLMOS et al., 2011; KRISHNAMURTI et al., 2012; SUNIKKA-BLANK et al., 2012; BOYLE et al., 2013; FENSEL et al., 2013; KAUFMANN; KÜNZL; LOOCK, 2013; RIXEN; WEIGAND, 2013; AKETI; SEN, 2014; JARAMILLO; CARDONA; HENAO, 2014; MARQUES; BENTO; COSTA, 2014; PARK; KIM; KIM, 2014; TORRII1, 2014; BALTA-OZKAN; AMERIGHI; BOTELER, 2014; BEGIER, 2014; CHOU et al., 2015; BALLO, 2015; BARNICOAT; DANSON, 2015; BUCHANAN et al., 2016;

Expectativa de CAMPILLO et al., 2016; ESPLUGA et al., 2016; MICHAELS; PARAG, 2016; RAIMI CARRICO, 2016; VASSILEVA; CAMPILLO, 2016; CHEN; XU; ARPAN, 2017; MOSER, 2017;

ganho 2018; DÜSTEGÖR et al., 2018; ALKAWSI; ALI; ALGHUSHAMI, 2018; LIU; MUKHEIBIR, 2018; MAH et al., 2018; ALSKAIF et al., 2018; MONTGINOUL; VESTIER, 2018; PAIHO et al

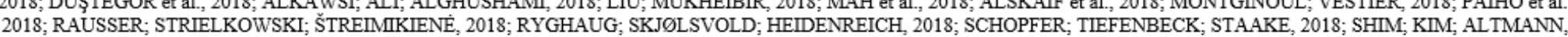
2018; SIL VAST et al., 2018; WEMYSS et al., 2018; BIGERNA et a1., 2018; BUGDEN; STEDMAN, 2019; GUO; LAM; LI, 2019; KIGUCHI et a1., 2019; YANG et al., 2019; CAHN; KATZ; GHERMANDI, 2020; CHAWLA; KOWALSKA-PYZALSKA; SII VEIRA, 2020; CHOI et al., 2020; VAN DE KAA et al., 2020)

(OITRA et al., 2013, BALTA

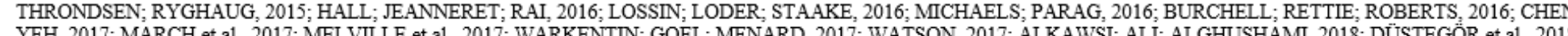
35 (2018;

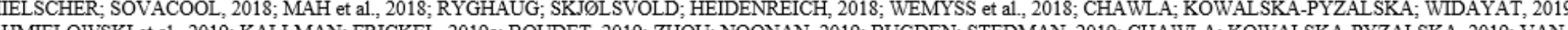
DE KAA I 2020;

DE

Histórico

Pessoal

Favorável

GERPOTT; PAUKERT, 2013; STEWART et al., 2013; CHOU; YUTAMI, 2014; DAVIES et al., 2014; MATSCHOSS; KAHMA; HEISKANEN, 2015; CHOU et a1., 2015; WATSON, 2017;

18 2019; SAREEN; ROMMETVEIT, 2019; BROWN; MARKUSSON, 2019; CHAWLA; KOWALSKA-PYZALSKA; WIDAYAT, 2019; CHAWLA; KOWALSKA-PYZALSKA; ORALHAN, 2020; CHAWLA; KOWALSKA-PYZALSKA; SILVEIRA, 2020)

Atitude em (KRISHNAMURTI et a1, 2012. CHOU; YUTAMI, 2014-PARK- KIM- KIM 2014; CHOU et a1 2015; MATSCHOSS- KAHMA- HEISKANEN, 2015; NACHREINER et al, 2015; direç̃ a

8

Caracteristicas CHAWLA; KOWALSKA-PYZALSKA; WIDAYAT, 2019; CHAWLA; KOWALSKA-PYZALSKA; ORALHAN, 2020)

(

demográficas

11

Motivação

politica ORALHAN, 2020)

(HESS, 2014; HESS; COLEY, 2014; CHEN; XU; ARPAN, 2017; KALLMAN; FRICKEL, 2019a)

Rede elétrica

(LOSSIN; LODER; STAAKE, 2016; MICHAELS; PARAG, 2016; ALKAWSI; ALI; ALGHUSHAMI, 2018)

(HARTWAY; PRICE; WOO 1999. BOYLE et al., 2013; BURYK et al., 2015; LOSSIN; LODER- STAAKE, 2016; WARKENTIN; GOEL; MENARD, 2017; ZHOU; BROWN, 2017; JEGEN; desconto SILVEIRA, 2020) 
Em relação a dimensão tecnologia, o benefício para aceitação mais mencionado na literatura é a utilidade percebida, construto tradicionalmente utilizado em surveys que utilizam como base o Modelo de Aceitação de Tecnologia (CHOU; YUTAMI, 2014; CHEN; XU; ARPAN, 2017). Outros dois benefícios também frequentemente mencionados são a energia limpa e a facilidade de uso percebido. $A$ respeito da energia limpa, a aceitação do medidor é influenciada de maneira positiva porque os medidores são percerbidos como uma forma de habilitar a rede elétrica para o uso de energias de fontes renováveis (MOGLES et al., 2017; ALKAWSI; ALI; ALGHUSHAMI, 2018; DÜŞTEGÖR et al., 2018; BUGDEN; STEDMAN, 2019). Já a facilidade de uso percebida é tradicionalmente utilizada em surveys que com a estrutura proposta no Modelo de Aceitação de Tecnologia (CHOU; YUTAMI, 2014; PARK; KIM; KIM, 2014; CHOU et al., 2015). A facilidade de uso percebida também está associada à familiaridade do usuário com o produto, indicando que produtos mais familiares ao usuário, são considerados mais fáceis de se utilizar e tendem a ser mais aceitos (BUGDEN; STEDMAN, 2019). Outros benefícios menos relatados são a integração com outras tecnologias e o abandono da opção de utilização de geração de energia de fonte nuclear.

Os benefícios associados à dimensão pessoas foram os mais frequentes na literatura, atingindo um total de 234 citações. Entre estes, a possibilidade de melhor gerenciar o consumo por meio do feedback é um dos fatores mais mencionados na literatura ao se pesquisar a aceitação de medidores inteligentes(DARBY, 2010). Apesar dessa importância, não é o medidor que fornece a visualização dos dados de consumo, mas sim uma tela (KRISHNAMURTI et al., 2012), ou mesmo um aplicativo de dispositivo móvel (FETTERMANN et al., 2020a, 2020b). Contudo, a importância do feedback para a mudança de hábitos do cliente é considerado um fator essencial para a aceitação de medidores inteligentes (KAUFMANN; KÜNZEL; LOOCK, 2013; CHOU; YUTAMI, 2014; CHEN; XU; ARPAN, 2017; MOGLES et al., 2017). A preocupação com as alterações climáticas (eco-preocupação) é outro fator importante na aceitação de tecnologias de energias sustentáveis, como o caso do medidor inteligente (CHEN; XU; ARPAN, 2017; BUGDEN; STEDMAN, 2019). Nesse sentido, as pessoas que se preocupam mais com o meio ambiente estariam mais dispostas a aceitar os medidores inteligentes (HUIJTS; MOLIN; STEG, 2012). A 
expectativa dos consumidores em ter ganho financeiro também tem se mostrado um dos principais fatores positivos, com vários estudos mostrando a relevância deste fator tanto para medidores de energia elétrica (e.g., KAUFMANN; KÜNZEL; LOOCK, 2013; PARK; KIM; KIM, 2014; CHOU et al., 2015; CHEN; XU; ARPAN, 2017; BUGDEN; STEDMAN, 2019), como também para medidores de água (e.g.,MONTGINOUL; VESTIER, 2018). Outros benefícios mencionados na literatura associados à dimensão pessoas estão relacionados aos fatores sociais, histórico pessoal favorável e atitude em direção ao uso (Erro! Fonte de referência não encontrada.).

Os benefícios associados a dimensão lugar são os menos frequentes na literatura. Nesta dimensão, o principal benefício é o grupo das características demográficas. Este benefício é composto por itens tais como idade, gênero, renda mensal, nível de educação formal e tamanho da residência. Não há um consenso na literatura sobre a influência de características demográficas na aceitação de medidores (FETTERMANN et al., 2020b). Algumas surveys indicam que os fatores demográficos não apresentam efeito significativo na adoção e aceitação de medidores residenciais inteligentes (e.g., DÜŞTEGÖR et al., 2018; CHAWLA; KOWALSKA-PYZALSKA; WIDAYAT, 2019; KIGUCHI et al., 2019; KOWALSKAPYZALSKA; BYRKA, 2019; CHAWLA; KOWALSKA-PYZALSKA; ORALHAN, 2020). Apesar disso, resultados recentes indicam que medidores inteligentes seriam mais aceitos por consumidores com baixa renda, por trazer vantagens e reduzir o valor da conta de energia (HIELSCHER; SOVACOOL, 2018; FETTERMANN et al., 2020a). Em relação ao nível educacional também se verificam resultados contrastantes, como menores níveis de aceitação por usuários menos escolarizados (WATSON, 2017), ao mesmo tempo outro estudo indica uma maior aceitação por parte de usuários com menor nível de educação formal (CHAWLA; KOWALSKA-PYZALSKA, 2019). Há também a indicação de que idosos são mais resistentes a aceitar os medidores inteligentes (BARNICOAT; DANSON, 2015) e que as mulheres tendem a assumir o protagonismo em relação à interação com medidores inteligentes (DAVIES et al., 2014). Outros benefícios associados à dimensão pessoas mencionados são a motivação política e a motivação extrínseca ( 


\section{Tabela 2).}

Somente dois benefícios mencionados na literatura foram categorizados à dimensão processo: rede elétrica mais resiliente e programas de descontos. Uma rede elétrica mais resiliente contra blecautes e com menor número de fraudes é um benefício não apenas para as companhias de energia, mas também para os consumidores (DARBY, 2010; PAIHO et al., 2018; SILVAST et al., 2018; YANG et al., 2019). Programas de desconto são tarifas diferenciadas e incentivos financeiros que são oferecidos durante 0 processo de implementação dos medidores inteligentes (HARTWAY; PRICE; WOO, 1999; LOSSIN; LODER; STAAKE, 2016; MICHAELS; PARAG, 2016).

\subsubsection{Barreiras para aceitação de medidores inteligentes}

Foram identificadas 190 citações de fatores considerados como barreiras associados a implementação de medidores inteligentes (Tabela 3). Entre estes a dimensão tecnologia é a mais frequente, seguida pela do processo e do lugar. Não foram identificadas barreiras na literatura para a aceitação de medidores inteligentes associadas a dimensão pessoas. 
Tabela 3 - Fatores considerados como barreiras para aceitação de medidores inteligentes

\begin{tabular}{|c|c|c|c|}
\hline & Fator & Freq. & Citado em \\
\hline \multirow[t]{4}{*}{ 鼠 } & Falta de Familiaridade & 39 & 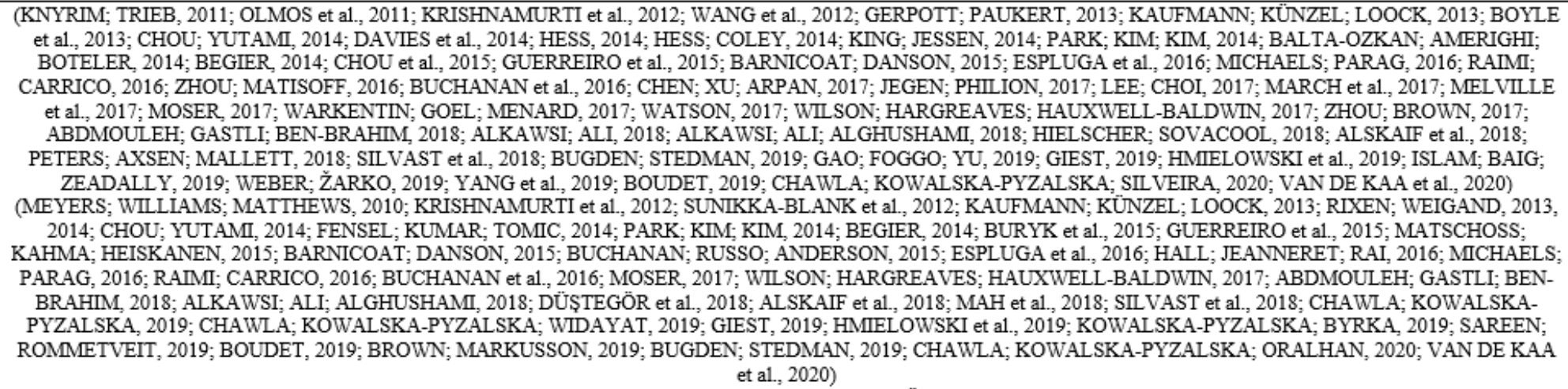 \\
\hline & Custos Associados & 25 & $\begin{array}{l}\text { (FARUQUI; HARRIS; HLEDIK, 2010; MEYERS; WILLIAMS; MATTHEWS, 2010; KAUFMANN; KÜNZEL; LOOCK, 2013; BOYLE et al., 2013; BEGIER, 2014; HESS, 2014; } \\
\text { CHOU; YUTAMI, 2014; GUERREIRO et al., 2015; MATSCHOSS; KAHMA; HEISKANEN, 2015; CHOU et al., 2015; ESPLUGA et a1., 2016; HALL; JEANNERET; RAI, 2016; } \\
\text { MICHAELS; PARAG, 2016; RAIMI; CARRICO, 2016; JEGEN; PHIIION, 2017; MOSER, 2017; CHEN; XU; ARPAN, 2017; CHEN; YEH, 2017; HIELSCHER; SOVACOOL, 2018; } \\
\text { PETERS; AXSEN; MALLETT, 2018; RAUSSER; STRIELKOWSKI; STREIMIKIENE, 2018; DÜŞTEGÖR et al., 2018; BOUDET, 2019; BUGDEN; STEDMAN, 2019; CHAWLA; } \\
\text { KOWALSKA-PYZALSKA, 2019) }\end{array}$ \\
\hline & Saúde ameaçada & 15 & $\begin{array}{l}\text { (BOYLE et al., 2013; PARK; KIM; KIM, 2014; HESS, 2014; HESS; COLEY, 2014; GUERREIRO et al., 2015; MICHAELS; PARAG, 2016; RAIMI; CARRICO, 2016; MOSER, } \\
\text { 2017; JEGEN; PHILION, 2017; PETERS; AXSEN; MALLETT, 2018; BOUDET, 2019; CHAWLA; KOWALSKA-PYZALSKA, 2019; CHAWLA; KOWALSKA-PYZALSKA; } \\
\text { WIDAYAT, 2019; CHAWLA; KOWALSKA-PYZALSKA; ORALHAN, 2020; CHAWLA; KOWALSKA-PYZALSKA; SILVEIRA, 2020) }\end{array}$ \\
\hline & Riscos gerais & 4 & (BEGIER, 2014; CHOU; YUTAMI, 2014; PARK; KIM; KIM, 2014; BOUDET, 2019) \\
\hline \multirow{3}{*}{ 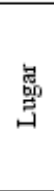 } & $\begin{array}{l}\text { Diminuição da liberdade } \\
\text { de escolha }\end{array}$ & 10 & $\begin{array}{l}\text { (BEGIER, 2014; HESS, 2014; HESS; COLEY, 2014; BUCHANAN et al., 2016; HALL; JEANNERET; RAI, 2016; HIELSCHER; SOVACOOL, 2018; PETERS; AXSEN; MALLETT, } \\
\text { 2018; CHAWLA; KOWALSKA-PYZALSKA, 2019; CHAWLA; KOWALSKA-PYZALSKA; WDAYAT, 2019; CHAWLA; KOWALSKA-PYZALSKA; ORALHAN, 2020) }\end{array}$ \\
\hline & $\begin{array}{l}\text { Baixo potencial de } \\
\text { economia / Fuel poverty }\end{array}$ & 4 & (DAVIES et al., 2014; BUCHANAN; RUSSO; ANDERSON, 2015; SILVAST et al., 2018; BROWN; MARKUSSON, 2019) \\
\hline & $\begin{array}{c}\text { Distância de grandes } \\
\text { centros }\end{array}$ & 2 & (BALTA-OZKAN; AMERIGHI; BOTELER, 2014; BOUDET, 2019) \\
\hline \multirow{3}{*}{$\begin{array}{l}\text { 总 } \\
\text { 怘 } \\
\text { 点 }\end{array}$} & Falta de confiança & 21 & $\begin{array}{l}\text { (BEGIER, 2014; MATSCHOSS; KAHMA; HEISKANEN, 2015; THRONDSEN; RYGHAUG, 2015; CHOU et al., 2015; MICHAELS; PARAG, 2016; RAIMI; CARRICO, 2016; } \\
\text { BUCHANAN et al., 2016; JEGEN; PHIIION, 2017; WARKENTIN; GOEL; MENARD, 2017; WILSON; HARGREAVES; HAUXWELL-BALDWIN, 2017; CHEN; XU; ARPAN, } \\
\text { 2017; ABDMOULEH; GASTLI; BEN-BRAHIM, 2018; ALKAWSI; ALI, 2018; MAH et a1., 2018; SHIM; KIM; ALTMANN, 2018; SAREEN; ROMMETVEIT, 2019; BOUDET, } \\
\text { 2019; BUGDEN; STEDMAN, 2019; CHAWLA; KOWALSKA-PYZALSKA; WIDAYAT, 2019; VAN DE KAA et a1., 2020; CHAWLA; KOWALSKA-PYZALSKA; ORALHAN, } \\
\text { 2020) }\end{array}$ \\
\hline & $\begin{array}{l}\text { Uso indevido de } \\
\text { informações }\end{array}$ & 18 & $\begin{array}{l}\text { (KNYRIM; TRIEB, 2011; BEGIER, 2014; KING; JESSEN, 2014; CHOU; YUTAMI, 2014; HESS, 2014; MICHAELS; PARAG, 2016; WARKENTIN; GOEL; MENARD, 2017; } \\
\text { WILSON; HARGREAVES; HAUXWELL-BALDWIN, 2017; BUGDEN; STEDMAN, 2019; WEBER; ŻARK, 2019; YANG et al., 2019; CHAWLA; KOWALSKA-PYZALSKA, } \\
\text { 2019; CHAWLA; KOWALSKA-PYZALSKA; WIDAYAT, 2019; GIEST, 2019; GUO; LAM; LI, 2019; KALLMAN; FRICKEL, 2019a; CHAWLA; KOWALSKA-PYZALSKA; } \\
\text { ORALHAN, 2020) }\end{array}$ \\
\hline & $\begin{array}{l}\text { Instalação em locais } \\
\text { pouco apropriados }\end{array}$ & 2 & (DAVIES et al., 2014; SILVAST et al., 2018) \\
\hline
\end{tabular}

Revista Produção Online. Florianópolis, SC, v. 21, n. 1, p. 131-156, 2021 
Entre as barreiras associadas a dimensão tecnologia foram identificadas 133 citações, sendo a dimensão de barreiras mais reportada na literatura (Tabela 3).

Um dos principais fatores que mais prejudicam a aceitação dos medidores inteligentes associados à dimensão tecnologia é a sensação de segurança ameaçada, com 50 citações na literatura. Este fator abrange diversos tópicos, como problemas de privacidade, com a possibilidade de a rotina da casa, por meio do perfil de uso de energia, estar sendo observada por companhias de energia ou por terceiros (CHEN; XU; ARPAN, 2017; BOUDET, 2019; BUGDEN; STEDMAN, 2019) e até mesmo pelos próprios integrantes da família, monitorando uns aos outros (YANG et al., 2019). Outro problema reportado é o de hackers acessarem o sistema, que resultaria em roubo de dados, venda de informações particulares e manipulação dos sistemas conectados ao medidor (CHOU; YUTAMI, 2014; WUNDERLICH; VEIT; SARKER, 2019). Além da invasão virtual, outro problema de segurança mencionado é que ao saber a rotina da casa, pessoas poderiam se aproveitar disso para invadir fisicamente as residências (CHOU; YUTAMI, 2014; CHEN; XU; ARPAN, 2017; BUGDEN; STEDMAN, 2019). A falta de familiaridade, que também provoca uma percepção de que os medidores sejam muito complexos, pode acabar influenciando negativamente a aceitação, como indicam alguns estudos (e.g., $\mathrm{CHOU}$ et al., 2015; DÜŞTEGÖR et al., 2018; BOUDET, 2019). Entre os custos à implementação de medidores inteligentes residenciais destacam-se o medo de ocorrência de faturas mais altas devido a maior acuracidade dos medidores inteligentes se comparados aos medidores mecânicos (PETERS; AXSEN; MALLETT, 2018; BUGDEN; STEDMAN, 2019). Também são considerados os custos imediatos de instalação e aquisição, quando o medidor não é cedido (PETERS; AXSEN; MALLETT, 2018; BUGDEN; STEDMAN, 2019) e o longo tempo para o payback do equipamento (BOUDET, 2019). Também são mencionados potenciais danos à saúde associados aos medidores inteligentes, decorrentes da emissão de ondas eletromagnéticas (PARK; KIM; KIM, 2014; MOSER, 2017; PETERS; AXSEN; MALLETT, 2018). A percepção geral de risco também é mencionada como uma barreira para aceitação de medidores inteligentes, relacionada à tecnologia.

Em relação a dimensão lugar, a principal barreira é a diminuição da liberdade de escolha, que ocorre quando a implementação dos medidores 
inteligentes apresenta um caráter obrigatório. Um trabalho realizado na Polônia mostrou que a maioria das pessoas gostaria que houvesse uma chance de escolha entre instalar ou não o medidor (CHAWLA; KOWALSKA-PYZALSKA, 2019), resultado semelhante ao encontrado na Indonésia (CHAWLA; KOWALSKAPYZALSKA; WIDAYAT, 2019) e na Turquia (CHAWLA; KOWALSKA-PYZALSKA; ORALHAN, 2020). Em locais do Canadá e dos Estados Unidos, houve contestação judicial da obrigatoriedade de implementação, sob a alegação de que os medidores ofereceriam riscos à saúde (HESS, 2014; HESS; COLEY, 2014). Outras barreiras relacionadas ao lugar são o pouco potencial de economia em casas que já tem um padrão baixo de consumo e a distância de grandes centros.

A dimensão processo agrupa quatro diferentes barreiras para a implementação dos medidores inteligentes. A aceitação de medidores inteligentes pode ser afetada negativamente pela falta de confiança dos usuários nos fornecedores de energia (e.g., BUCHANAN et al., 2016; JEGEN; PHILION, 2017). Esta falta de confiança pode ser ocasionada pela ausência de uma comunicação efetiva com os consumidores durante a implementação dos medidores inteligentes. Outro ponto que gera desconfiança é a falta de transparência no gerenciamento das informações dos usuários (JEGEN; PHILION, 2017). Outra barreira consiste no medo de que as informações de padrão de consumo dos usuários sejam utilizadas de forma indevida pelas companhias, principalmente sendo compartilhadas com terceiros (BUGDEN; STEDMAN, 2019). Outra barreira relatada em relação ao processo de implementação de medidores inteligentes é a instalação do medidor em locais da casa com pouco tráfego de pessoas.

\section{CONSIDERAÇÕES}

O presente trabalho apresenta uma revisão sistemática da literatura baseada no método PRISMA (LIBERATI et al., 2009) para a identificação de fatores que afetam a aceitação de medidores inteligentes por parte de clientes residenciais. A literatura analisada evidencia o baixo conhecimento sobre a aceitação de medidores inteligentes por parte de consumidores de países em desenvolvimento, tal como os BRICS (Brasil, Rússia, Índia, China e África do Sul). Apesar destes países liderarem 
mundialmente em expansão de capacidade para fontes renováveis e capacidade instalada para fontes renováveis (REN21, 2020), somente foi identificado um estudo realizado nestes países (BOOYSEN; VISSER; BURGER, 2019). Ainda que a realidade brasileira não seja igual a de nenhum outro país, o presente trabalho contribuiu reunindo fatores, construtos e teorias que podem ser utilizados como ponto de partida para a pesquisa de aceitação de medidores inteligentes no Brasil.

É interessante ressaltar que um dos principais fatores apontados para a diminuição da aceitação de medidores é a falta de familiaridade dos consumidores com os medidores inteligentes. Logo, apresentar ao público o que é o medidor inteligente, por meio desta pesquisa, já fará com que se diminua a barreira para aceitação. Futuros trabalhos podem usar os resultados desta pesquisa como base para incorporar funções ao medidor inteligente e aprimorar os processos de implementação a fim de potencializar a aceitação entre os consumidores e por consequência avançar em direção às metas de economia de energia elétrica.

\section{AGRADECIMENTOS}

Esta pesquisa é suportada pelo Conselho Nacional de Desenvolvimento Científico e Tecnológico (CNPq) [303936-2019-3 e bolsa de estudos] e Coordenação de Aperfeiçoamento de Pessoal de Nível Superior (CAPES) [Finance Code 001].

\section{REFERÊNCIAS}

ABDMOULEH, Z.; GASTLI, A.; BEN-BRAHIM, L. Survey about public perception regarding smart grid, energy efficiency \& renewable energies applications in Qatar. Renewable and Sustainable Energy Reviews, v. 82, p. 168-175, 2018. https://doi.org/10.1016/i.rser.2017.09.023

AKETI, P.; SEN, S. Modeling demand response and economic impact of advanced and smart metering. Energy Systems, v. 5, n. 3, p. 583-606, 2014. https://doi.org/10.1007/s12667-013-0113-1

ALKAWSI, G. A.; ALI, N. B. A systematic review of individuals' acceptance of IOT-based technologies. International Journal of Engineering and Technology, v. 7, n. 4.35, p. 136142, 2018. https://doi.org/10.14419/ijet.v7i4.35.22342

ALKAWSI, G. A.; ALI, N. B.; ALGHUSHAMI, A. Toward understanding individuals' acceptance of internet of things -based services: Developing an instrument to measure the 
acceptance of smart meters. Journal of Theoretical and Applied Information

Technology, v. 96, n. 13, p. 4265-4281, 2018.

ALSKAIF, T. et al. Gamification-based framework for engagement of residential customers in energy applications. Energy Research and Social Science, v. 44, p. 187-195, 2018.

https://doi.org/10.1016/j.erss.2018.04.043

ARNOLD, O. et al. Capturing the Environmental Impact of Individual Lifestyles: Evidence of the Criterion Validity of the General Ecological Behavior Scale. Environment and Behavior, v. 50, n. 3, p. 350-372, 2018. https://doi.org/10.1177/0013916517701796

AVANCINI, D. B. et al. Energy meters evolution in smart grids: A review. Journal of Cleaner Production, v. 217, p. 702-715, 2019. https://doi.org/10.1016/i.jclepro.2019.01.229

AYODELE, O. A.; CHANG-RICHARDS, A.; GONZÁLEZ, V. Factors Affecting Workforce Turnover in the Construction Sector: A Systematic Review. Journal of Construction Engineering and Management, v. 146, n. 2, p. 03119010, 2020.

https://doi.org/10.1061/(ASCE)CO.1943-7862.0001725

BALLO, I. F. Imagining energy futures: Sociotechnical imaginaries of the future Smart Grid in Norway. Energy Research and Social Science, v. 9, p. 9-20, 2015.

https://doi.org/10.1016/j.erss.2015.08.015

BALTA-OZKAN, N.; AMERIGHI, O.; BOTELER, B. A comparison of consumer perceptions towards smart homes in the UK, Germany and Italy: reflections for policy and future research. Technology Analysis and Strategic Management, v. 26, n. 10, p. 1176-1195, 2014. https://doi.org/10.1080/09537325.2014.975788

BARBOUR, E.; GONZÁLEZ, M. C. Projecting battery adoption in the prosumer era. Applied Energy, v. 215, p. 356-370, 2018. https://doi.org/10.1016/j.apenergy.2018.01.056

BARNICOAT, G.; DANSON, M. The ageing population and smart metering: A field study of householders' attitudes and behaviours towards energy use in Scotland. Energy Research and Social Science, v. 9, n. September 2015, p. 107-115, 2015.

https://doi.org/10.1016/j.erss.2015.08.020

BEAL, C. D. et al. Identifying and understanding the drivers of high water consumption in remote Australian Aboriginal and Torres Strait Island communities. Journal of Cleaner Production, v. 172, p. 2425-2434, 2018. https://doi.org/10.1016/j.jclepro.2017.11.168

BEAL, C. D.; FLYNN, J. Toward the digital water age: Survey and case studies of Australian water utility smart-metering programs. Utilities Policy, v. 32, p. 29-37, 2015.

https://doi.org/10.1016/j.jup.2014.12.006

BEAL, C. D.; GURUNG, T. R.; STEWART, R. A. Modelling the impacts of water efficient technologies on energy intensive water systems in remote and isolated communities. Clean Technologies and Environmental Policy, v. 18, n. 6, p. 1713-1723, 2016.

https://doi.org/10.1007/s10098-016-1241-9

BEGIER, B. Effective cooperation with energy consumers: An example of an ethical approach to introduce an innovative solution. Journal of Information, Communication and Ethics in Society, v. 12, n. 2, p. 107-121, 2014. https://doi.org/10.1108/JICES-07-2013- 
0021

BERTOLDO, R.; POUMADÈRE, M.; RODRIGUES JR, L. C. When meters start to talk: The public's encounter with smart meters in France. Energy Research and Social Science, v. 9, p. 146-156, 2015. https://doi.org/10.1016/i.erss.2015.08.014

BIGERNA, S. et al. A new unified approach to evaluate economic acceptance towards main green technologies using the meta-analysis. Journal of Cleaner Production, v. 167, p. 1251-1262, 2018. https://doi.org/10.1016/j.jclepro.2017.07.188

BOAIT, P. J. et al. Making legacy thermal storage heating fit for the smart grid. Energy and Buildings, v. 138, p. 630-640, 2017. https://doi.org/10.1016/i.enbuild.2016.12.053

BOOYSEN, M. J.; VISSER, M.; BURGER, R. Temporal case study of household behavioural response to Cape Town's "Day Zero" using smart meter data. Water Research, v. 149, p. 414-420, 2019. https://doi.org/10.1016/j.watres.2018.11.035

BOUDET, H. S. Public perceptions of and responses to new energy technologies. Nature Energy, v. 4, n. 6, p. 446-455, 2019. https://doi.org/10.1038/s41560-019-0399-x

BOYLE, T. et al. Intelligent metering for urban water: A review. Water, v. 5, n. 3, p. 10521081, 2013. https://doi.org/10.3390/w5031052

BROWN, C. J.; MARKUSSON, N. The responses of older adults to smart energy monitors. Energy Policy, v. 130, p. 218-226, 2019. https://doi.org/10.1016/i.enpol.2019.03.063

BUCHANAN, K. et al. The British public's perception of the UK smart metering initiative: Threats and opportunities. Energy Policy, v. 91, p. 87-97, 2016.

https://doi.org/10.1016/i.enpol.2016.01.003

BUCHANAN, K.; RUSSO, R.; ANDERSON, B. The question of energy reduction: The problem(s) with feedback. Energy Policy, v. 77, p. 89-96, 2015.

https://doi.org/10.1016/j.enpol.2014.12.008

BUGDEN, D.; STEDMAN, R. A synthetic view of acceptance and engagement with smart meters in the United States. Energy Research and Social Science, v. 47, n. January 2019, p. 137-145, 2019. https://doi.org/10.1016/j.erss.2018.08.025

BURCHELL, K.; RETTIE, R.; ROBERTS, T. C. Householder engagement with energy consumption feedback: The role of community action and communications. Energy Policy, v. 88, p. 178-186, 2016. https://doi.org/10.1016/i.enpol.2015.10.019

BURYK, S. et al. Investigating preferences for dynamic electricity tariffs: The effect of environmental and system benefit disclosure. Energy Policy, v. 80, p. 190-195, 2015. https://doi.org/10.1016/j.enpol.2015.01.030

CAHN, A.; KATZ, D.; GHERMANDI, A. Analyzing Water Customer Preferences for Online Feedback Technologies in Israel: A Prototype Study. Journal of Water Resources Planning and Management, v. 146, n. 4, p. 06020002, 2020.

https://doi.org/10.1061/(ASCE)WR.1943-5452.0001179

CAMPILLO, J. et al. Is real-time electricity pricing suitable for residential users without demand-side management? Energy, v. 109, p. 310-325, 2016. 
https://doi.org/10.1016/j.energy.2016.04.105

CHABÉ-FERRET, S. et al. Can we nudge farmers into saving water? Evidence from a randomised experiment. European Review of Agricultural Economics, v. 46, n. 3, p. 393416, 2019. https://doi.org/10.1093/erae/jbz022

CHAWLA, Y.; KOWALSKA-PYZALSKA, A. Public Awareness and Consumer Acceptance of Smart Meters among Polish Social Media Users. Energies, v. 12, n. 14, p. 2759, 2019. https://doi.org/10.3390/en12142759

CHAWLA, Y.; KOWALSKA-PYZALSKA, A.; ORALHAN, B. Attitudes and opinions of social media users towards smart meters' rollout in Turkey. Energies, v. 13, n. 3, p. 732, 2020. https://doi.org/10.3390/en13030732

CHAWLA, Y.; KOWALSKA-PYZALSKA, A.; SILVEIRA, P. D. Marketing and communications channels for diffusion of electricity smart meters in Portugal. Telematics and Informatics, v. 50, p. 101385, 2020. https://doi.org/10.1016/j.tele.2020.101385

CHAWLA, Y.; KOWALSKA-PYZALSKA, A.; WIDAYAT, W. Consumer Willingness and Acceptance of Smart Meters in Indonesia. Resources, v. 8, n. 4, p. 177, 2019.

https://doi.org/10.3390/resources8040177

CHEN, C.; XU, X.; ARPAN, L. Between the technology acceptance model and sustainable energy technology acceptance model: Investigating smart meter acceptance in the United States. Energy Research and Social Science, v. 25, p. 93-104, 2017. https://doi.org/10.1016/i.erss.2016.12.011

CHEN, K.-Y.; YEH, C.-F. Factors affecting adoption of smart meters in the post-Fukushima era in Taiwan: an extended protection motivation theory perspective. Behaviour and Information Technology, v. 36, n. 9, p. 955-969, 2017. https://doi.org/10.1080/0144929X.2017.1317363

CHEN, V. L.; DELMAS, M. A.; KAISER, W. J. Real-time, appliance-level electricity use feedback system: How to engage users? Energy and Buildings, v. 70, p. 455-462, 2014. https://doi.org/10.1016/j.enbuild.2013.11.069

CHOI, D. G. et al. Why Have Voluntary Time-of-Use Tariffs Fallen Short in the Residential Sector? Production and Operations Management, v. 29, n. 3, p. 617-642, 2020. https://doi.org/10.1111/poms.13126

$\mathrm{CHOU}$, J.-S. et al. Cross-country review of smart grid adoption in residential buildings. Renewable and Sustainable Energy Reviews, v. 48, p. 192-213, 2015. https://doi.org/10.1016/i.rser.2015.03.055

CHOU, J.-S.; YUTAMI, I. G. A. N. Smart meter adoption and deployment strategy for residential buildings in Indonesia. Applied Energy, v. 128, p. 336-349, 2014. https://doi.org/10.1016/i.apenergy.2014.04.083

DARBY, S. Smart metering: what potential for householder engagement? Building Research and Information, v. 38, n. 5, p. 442-457, 2010. https://doi.org/10.1080/09613218.2010.492660 
DAVIES, K. et al. Water-saving impacts of Smart Meter technology: An empirical 5 year, whole-of-community study in Sydney, Australia. Water Resources Research, v. 50, n. 9, p. 7348-7358, set. 2014. https://doi.org/10.1002/2014WR015812

DAVIS, F. D.; BAGOZZI, R. P.; WARSHAW, P. R. User Acceptance of Computer Technology: A Comparison of Two Theoretical Models. Management Science, v. 35, n. 8, p. 982-1003, 1989. https://doi.org/10.1287/mnsc.35.8.982

DEGEN, H.; MOSTASHARI, A.; MAURER, M. A sociotechnical systems model for smart-grid adoption in mid-sized urban environments. International Journal of Energy Technology and Policy, v. 10, n. 1, p. 1-20, 2014. https://doi.org/10.1504//JETP.2014.065034

DÜŞTEGÖR, D. et al. A smarter electricity grid for the Eastern Province of Saudi Arabia: Perceptions and policy implications. Utilities Policy, v. 50, p. 26-39, 2018.

https://doi.org/10.1016/i.jup.2017.09.009

DYSON, M. E. H. et al. Using smart meter data to estimate demand response potential, with application to solar energy integration. Energy Policy, v. 73, p. 607-619, 2014.

https://doi.org/10.1016/i.enpol.2014.05.053

ELLABBAN, O.; ABU-RUB, H. Smart grid customers' acceptance and engagement: An overview. Renewable and Sustainable Energy Reviews, v. 65, p. 1285-1298, 2016. https://doi.org/10.1016/j.rser.2016.06.021

ESPLUGA, J. et al. How to address citizens' practices and policies on sustainability? A consultative tool for brokering policy-related knowledge between the worlds of policymaking and everyday citizens' life. Evidence and Policy, v. 12, n. 3, p. 381-404, 2016.

https://doi.org/10.1332/174426416X14738559545991

EUROPEAN UNION. Directive of 2009/72/EC of the European Parliament and of the Council of 13 July 2009 Concerning Common Rules for the Internal Market in Electricity and Repealing Directive 2003/54/EC. Official Journal of the European Union, v. L211, n. August, p. L 211/55-L 211/93, 2009. Disponível em: https://eurlex.europa.eu/LexUriServ/LexUriServ.do?uri=OJ:L:2009:211:0055:0093:EN:PDF.

FARUQUI, A.; HARRIS, D.; HLEDIK, R. Unlocking the $€ 53$ billion savings from smart meters in the EU: How increasing the adoption of dynamic tariffs could make or break the EU's smart grid investment. Energy Policy, v. 38, n. 10, p. 6222-6231, 2010.

https://doi.org/10.1016/i.enpol.2010.06.010

FENSEL, A. et al. SESAME-S: Semantic smart home system for energy efficiency. Informatik-Spektrum, v. 36, n. 1, p. 46-57, 2013. https://doi.org/10.1007/s00287-012-0665$\underline{9}$

FENSEL, A.; KUMAR, V.; TOMIC, S. D. K. End-user interfaces for energy-efficient semantically enabled smart homes. Energy Efficiency, v. 7, n. 4, p. 655-675, 2014. https://doi.org/10.1007/s12053-013-9246-2

FETTERMANN, D. C. et al. Configuration of a smart meter for Brazilian customers. Energy Policy, v. 139, p. 111309, 2020a. https://doi.org/10.1016/i.enpol.2020.111309

FETTERMANN, D. C. et al. Getting smarter about household energy: the who and what of 
demand for smart meters. Building Research and Information, 2020b.

https://doi.org/10.1080/09613218.2020.1807896

$\mathrm{GAO}, \mathrm{X}$. et al. Systematic review on the implementation methodologies of inherent safety in chemical process. Journal of Loss Prevention in the Process Industries, v. 65, p. 104092, 2020. https://doi.org/10.1016/i.jp.2020.104092

GAO, Y.; FOGGO, B.; YU, N. A Physically Inspired Data-Driven Model for Electricity Theft Detection With Smart Meter Data. IEEE Transactions on Industrial Informatics, v. 15, n. 9, p. 5076-5088, 2019. https://doi.org/10.1109/TII.2019.2898171

GARRAB, A.; BOUALLEGUE, A.; BOUALLEGUE, R. An agent based fuzzy control for smart home energy management in smart grid environment. International Journal of Renewable Energy Research, v. 7, n. 2, p. 599-612, 2017.

GERPOTT, T. J.; PAUKERT, M. Determinants of willingness to pay for smart meters: An empirical analysis of household customers in Germany. Energy Policy, v. 61, p. 483-495, 2013. https://doi.org/10.1016/i.enpol.2013.06.012

GIACOMIN, J.; BERTOLA, D. Human emotional response to energy visualisations. International Journal of Industrial Ergonomics, v. 42, n. 6, p. 542-552, 2012. https://doi.org/10.1016/j.ergon.2012.07.006

GIEST, S. Making energy personal: Policy coordination challenges in UK smart meter implementation. Journal of Public Policy, v. 40, n. 4, p. 553-572, 2019.

https://doi.org/10.1017/S0143814X19000163

GRANDCLÉMENT, C.; NADAII, A. Devising the consumer of the competitive electricity market: the mundane meter, the unbundling doctrine, and the re-bundling of choice. Journal of Cultural Economy, v. 11, n. 5, p. 440-457, 2018.

https://doi.org/10.1080/17530350.2018.1488269

GUERREIRO, S. et al. Making energy visible: sociopsychological aspects associated with the use of smart meters. Energy Efficiency, v. 8, n. 6, p. 1149-1167, 2015.

https://doi.org/10.1007/s12053-015-9344-4

GUO, P.; LAM, J. C. K.; LI, V. O. K. Drivers of domestic electricity users' price responsiveness: A novel machine learning approach. Applied Energy, v. 235, p. 900-913, 2019. https://doi.org/10.1016/i.apenergy.2018.11.014

HALL, N. L.; JEANNERET, T. D.; RAI, A. Cost-reflective electricity pricing: Consumer preferences and perceptions. Energy Policy, v. 95, p. 62-72, 2016.

https://doi.org/10.1016/i.enpol.2016.04.042

HARTWAY, R.; PRICE, S.; WOO, C. K. Smart meter, customer choice and profitable time-ofuse rate option. Energy, v. 24, n. 10, p. 895-903, 1999. https://doi.org/10.1016/S0360-

5442(99)00040-7

HESS, D. J. Smart meters and public acceptance: Comparative analysis and governance implications. Health, Risk and Society, v. 16, n. 3, p. 243-258, 2014.

https://doi.org/10.1080/13698575.2014.911821 
HESS, D. J.; COLEY, J. S. Wireless smart meters and public acceptance: The environment, limited choices, and precautionary politics. Public Understanding of Science, v. 23, n. 6, p. 688-702, 2014. https://doi.org/10.1177/0963662512464936

HIELSCHER, S.; SOVACOOL, B. K. Contested smart and low-carbon energy futures: Media discourses of smart meters in the United Kingdom. Journal of Cleaner Production, v. 195, p. 978-990, 2018. https://doi.org/10.1016/j.jclepro.2018.05.227

HMIELOWSKI, J. D. et al. The social dimensions of smart meters in the United States: Demographics, privacy, and technology readiness. Energy Research and Social Science, v. 55, p. 189-197, 2019. https://doi.org/10.1016/j.erss.2019.05.003

HOSSAIN, M. S. et al. Role of smart grid in renewable energy: An overview. Renewable and Sustainable Energy Reviews, v. 60, p. 1168-1184, 2016.

https://doi.org/10.1016/j.rser.2015.09.098

HU, C. et al. CP_ABSC: An attribute-based signcryption scheme to secure multicast communications in smart grids. Mathematical Foundations of Computing, v. 1, n. 1, p. 77100, 2018.

HUIJTS, N. M. A.; MOLIN, E. J. E.; STEG, L. Psychological factors influencing sustainable energy technology acceptance: A review-based comprehensive framework. Renewable and Sustainable Energy Reviews, v. 16, n. 1, p. 525-531, 2012.

https://doi.org/10.1016/j.rser.2011.08.018

ISLAM, S. N.; BAIG, Z.; ZEADALLY, S. Physical Layer Security for the Smart Grid: Vulnerabilities, Threats, and Countermeasures. IEEE Transactions on Industrial Informatics, v. 15, n. 12, p. 6522-6530, 2019. https://doi.org/10.1109/TII.2019.2931436

JARAMILLO, N. C.; CARDONA, C. J. F.; HENAO, J. D. V. Smart meters adoption: recent advances and future trends. DYNA, v. 81, n. 183, p. 221-230, 24 fev. 2014. Disponível em: http://www.scopus.com/inward/record.url?eid=2-s2.0-84894593003\&partnerID=MN8TOARS. https://doi.org/10.15446/dyna.v81n183.38148

JEGEN, M.; PHILION, X. D. Power and smart meters: A political perspective on the social acceptance of energy projects. Canadian Public Administration, v. 60, n. 1, p. 68-88, 2017. https://doi.org/10.1111/capa.12202

KALLMAN, M. E.; FRICKEL, S. Power to the people: industrial transition movements and energy populism. Environmental Sociology, v. 5, n. 3, p. 255-268, 2019a.

https://doi.org/10.1080/23251042.2018.1531497

KALLMAN, M. E.; FRICKEL, S. Nested logics and smart meter adoption: Institutional processes and organizational change in the diffusion of smart meters in the United States. Energy Research and Social Science, v. 57, n. November 2019, p. 101249, 2019b. https://doi.org/10.1016/i.erss.2019.101249

KAUFMANN, S.; KÜNZEL, K.; LOOCK, M. Customer value of smart metering: Explorative evidence from a choice-based conjoint study in Switzerland. Energy Policy, v. 53, p. 229239, 2013. https://doi.org/10.1016/i.enpol.2012.10.072

KIGUCHI, Y. et al. Predicting intra-day load profiles under time-of-use tariffs using smart 
meter data. Energy, v. 173, p. 959-970, 2019. https://doi.org/10.1016/i.energy.2019.01.037

KING, N. J.; JESSEN, P. W. Smart metering systems and data sharing: Why getting a smart meter should also mean getting strong information privacy controls to manage data sharing. International Journal of Law and Information Technology, v. 22, n. 3, p. 215-253, 2014. https://doi.org/10.1093/ij|lit/eau001

KNYRIM, R.; TRIEB, G. Smart metering under EU data protection law. International Data Privacy Law, v. 1, n. 2, p. 121-128, 2011. https://doi.org/10.1093/idpl/ipr004

KOWALSKA-PYZALSKA, A.; BYRKA, K. Determinants of the willingness to energy monitoring by residential consumers: A case study in the city of Wroclaw in Poland. Energies, v. 12, n. 5, p. 907, 2019. https://doi.org/10.3390/en12050907

KRISHNAMURTI, T. et al. Preparing for smart grid technologies: A behavioral decision research approach to understanding consumer expectations about smart meters. Energy Policy, v. 41, p. 790-797, 2012. https://doi.org/10.1016/j.enpol.2011.11.047

KUMAR, A. Beyond technical smartness: Rethinking the development and implementation of sociotechnical smart grids in India. Energy Research and Social Science, v. 49, n. March 2019, p. 158-168, 2019. https://doi.org/10.1016/j.erss.2018.10.026

LEE, S.-H.; CHOI, K.-W. A study of smart meter security using Gaussian mixture model. Information, v. 20, n. 6, p. 3921-3926, 2017.

LIBERATI, A. et al. The PRISMA statement for reporting systematic reviews and metaanalyses of studies that evaluate health care interventions: Explanation and elaboration. PLoS Medicine, v. 6, n. 7, p. e1000100, 2009. https://doi.org/10.1371/journal.pmed.1000100

LIU, A.; MUKHEIBIR, P. Digital metering feedback and changes in water consumption - A review. Resources, Conservation and Recycling, v. 134, p. 136-148, 2018.

https://doi.org/10.1016/j.resconrec.2018.03.010

LOSSIN, F.; LODER, A.; STAAKE, T. Energy informatics for behavioral change: Increasing the participation rate in an IT-based energy conservation campaign using social norms and incentives. Computer Science - Research and Development, v. 31, n. 3, p. 149-155, 2016. https://doi.org/10.1007/s00450-014-0295-3

MAH, D. N. et al. Understanding undergraduate students' perceptions of dynamic pricing policies: An exploratory study of two pilot deliberative pollings (DPs) in Guangzhou, China and Kyoto, Japan. Journal of Cleaner Production, v. 202, p. 160-173, 2018.

https://doi.org/10.1016/j.jclepro.2018.07.255

MARCH, H. et al. Household Smart Water Metering in Spain: Insights from the Experience of Remote Meter Reading in Alicante. Sustainability, v. 9, n. 4, p. 582, 11 abr. 2017.

Disponível em: http://www.mdpi.com/2071-1050/9/4/582. https://doi.org/10.3390/su9040582

MARQUES, V.; BENTO, N.; COSTA, P. M. The "Smart Paradox": Stimulate the deployment of smart grids with effective regulatory instruments. Energy, v. 69, p. 96-103, 2014.

https://doi.org/10.1016/j.energy.2014.01.007

MATSCHOSS, K.; KAHMA, N.; HEISKANEN, E. Pioneering customers as change agents for 
new energy efficiency services-an empirical study in the Finnish electricity markets. Energy Efficiency, v. 8, n. 5, p. 827-843, 2015. https://doi.org/10.1007/s12053-014-9300-8

MELVILLE, E. et al. The electric commons: A qualitative study of community accountability. Energy Policy, v. 106, p. 12-21, 2017. https://doi.org/10.1016/i.enpol.2017.03.035

MEYERS, R. J.; WILLIAMS, E. D.; MATTHEWS, H. S. Scoping the potential of monitoring and control technologies to reduce energy use in homes. Energy and Buildings, v. 42, n. 5, p. 563-569, 2010. https://doi.org/10.1016/j.enbuild.2009.10.026

MICHAELS, L.; PARAG, Y. Motivations and barriers to integrating 'prosuming' services into the future decentralized electricity grid: Findings from Israel. Energy Research and Social Science, v. 21, p. 70-83, 2016. https://doi.org/10.1016/j.erss.2016.06.023

MOGLES, N. et al. How smart do smart meters need to be? Building and Environment, v. 125, p. 439-450, 2017. https://doi.org/10.1016/.j.buildenv.2017.09.008

MONTGINOUL, M.; VESTIER, A. Smart metering: A water-saving solution? Consider communication strategies and user perceptions first. Evidence from a French case study. Environmental Modelling and Software, v. 104, p. 188-198, 2018.

https://doi.org/10.1016/j.envsoft.2018.02.006

MORENO-BLANCO, D. et al. Technologies for monitoring lifestyle habits related to brain health: A systematic review. Sensors, v. 19, p. 4183, 2019.

https://doi.org/10.3390/s19194183

MOSER, C. The role of perceived control over appliances in the acceptance of electricity load-shifting programmes. Energy Efficiency, v. 10, n. 5, p. 1115-1127, 2017.

https://doi.org/10.1007/s12053-017-9508-5

MURTAGH, N. et al. Individual energy use and feedback in an office setting: A field trial. Energy Policy, v. 62, p. 717-728, 2013. https://doi.org/10.1016/i.enpol.2013.07.090

NACHREINER, M. et al. An analysis of smart metering information systems: A psychological model of self-regulated behavioural change. Energy Research and Social Science, v. 9, p. 85-97, 2015. https://doi.org/10.1016/j.erss.2015.08.016

NIKOLAIDIS, P.; CHATZIS, S.; POULLIKKAS, A. Life cycle cost analysis of electricity storage facilities in flexible power systems. International Journal of Sustainable Energy, v. 38, n. 8, p. 752-772, 2019. https://doi.org/10.1080/14786451.2019.1579815

NOVAK, J. et al. Integrating behavioural change and gamified incentive modelling for stimulating water saving. Environmental Modelling and Software, v. 102, p. 120-137, 2018. https://doi.org/10.1016/j.envsoft.2017.11.038

OLMOS, L. et al. Energy efficiency actions related to the rollout of smart meters for small consumers, application to the Austrian system. Energy, v. 36, n. 7, p. 4396-4409, 2011. https://doi.org/10.1016/j.energy.2011.04.003

OLTRA, C. et al. A qualitative study of users' engagement with real-time feedback from inhouse energy consumption displays. Energy Policy, v. 61, p. 788-792, 2013.

https://doi.org/10.1016/j.enpol.2013.06.127 
PAIHO, S. et al. Increasing flexibility of Finnish energy systems-A review of potential technologies and means. Sustainable Cities and Society, v. 43, p. 509-523, 2018. https://doi.org/10.1016/..scs.2018.09.015

PARK, C.-K.; KIM, H.-J.; KIM, Y.-S. A study of factors enhancing smart grid consumer engagement. Energy Policy, v. 72, p. 211-218, 2014.

https://doi.org/10.1016/j.enpol.2014.03.017

PETERS, D.; AXSEN, J.; MALLETT, A. The role of environmental framing in socio-political acceptance of smart grid: The case of British Columbia, Canada. Renewable and Sustainable Energy Reviews, v. 82, p. 1939-1951, 2018. https://doi.org/10.1016/j.rser.2017.06.020

RAIMI, K. T.; CARRICO, A. R. Understanding and beliefs about smart energy technology. Energy Research and Social Science, v. 12, p. 68-74, 2016. https://doi.org/10.1016/j.erss.2015.12.018

RAUSSER, G.; STRIELKOWSKI, W.; ŠTREIMIKIENĖ, D. Smart meters and household electricity consumption: A case study in Ireland. Energy and Environment, v. 29, n. 1, p. 131-146, 2018. https://doi.org/10.1177/0958305X17741385

REN21. Renewables 2020 Global Status Report. Paris: REN21 Secretariat, 2020.

RIXEN, M.; WEIGAND, J. Agent-based simulation of consumer demand for smart metering tariffs. International Journal of Innovation and Technology Management, v. 10, n. 5, p. 1340020, 2013. https://doi.org/10.1142/S0219877013400208

RIXEN, M.; WEIGAND, J. Agent-based simulation of policy induced diffusion of smart meters. Technological Forecasting and Social Change, v. 85, p. 153-167, 2014. https://doi.org/10.1016/i.techfore.2013.08.011

RYGHAUG, M.; SKJØLSVOLD, T. M.; HEIDENREICH, S. Creating energy citizenship through material participation. Social Studies of Science, v. 48, n. 2, p. 283-303, 2018. https://doi.org/10.1177/0306312718770286

SAREEN, S.; ROMMETVEIT, K. Smart gridlock? Challenging hegemonic framings of mitigation solutions and scalability. Environmental Research Letters, v. 14, n. 7, p. 075004, 2019. https://doi.org/10.1088/1748-9326/ab21e6

SCHOPFER, S.; TIEFENBECK, V.; STAAKE, T. Economic assessment of photovoltaic battery systems based on household load profiles. Applied Energy, v. 223, p. 229-248, 2018. https://doi.org/10.1016/i.apenergy.2018.03.185

SHIM, D.; KIM, S. W.; ALTMANN, J. Strategic management of residential electric services in the competitive market: Demand-oriented perspective. Energy and Environment, v. 29, n. 1, p. 49-66, 2018. https://doi.org/10.1177/0958305X17740234

SILVAST, A. et al. Who "uses" smart grids? The evolving nature of user representations in layered infrastructures. Sustainability, v. 10, n. 10, p. 3738, 2018.

https://doi.org/10.3390/su10103738 
SØNDERLUND, A. L. et al. Effectiveness of smart meter-based consumption feedback in curbing household water use: Knowns and unknowns. Journal of Water Resources

Planning and Management, v. 142, n. 12, p. 04016060, 2016.

https://doi.org/10.1061/(ASCE)WR.1943-5452.0000703

STEWART, R. A. et al. Showering behavioural response to alarming visual display monitors: Longitudinal mixed method study. Behaviour and Information Technology, v. 32, n. 7, p. 695-711, 2013. https://doi.org/10.1080/0144929X.2011.577195

SUNIKKA-BLANK, M. et al. Improving Energy Efficiency of Social Housing Areas: A Case Study of a Retrofit Achieving an "A" Energy Performance Rating in the UK. European

Planning Studies, v. 20, n. 1, p. 131-145, 2012.

https://doi.org/10.1080/09654313.2011.638494

THRONDSEN, W. What do experts talk about when they talk about users? Expectations and imagined users in the smart grid. Energy Efficiency, v. 10, n. 2, p. 283-297, 3 abr. 2017. Disponível em: http://link.springer.com/10.1007/s12053-016-9456-5.

https://doi.org/10.1007/s12053-016-9456-5

THRONDSEN, W.; RYGHAUG, M. Material participation and the smart grid: Exploring different modes of articulation. Energy Research and Social Science, v. 9, p. 157-165, 2015. https://doi.org/10.1016/j.erss.2015.08.012

TORRITI, J. People or machines? Assessing the impacts of smart meters and load controllers in Italian office spaces. Energy for Sustainable Development, v. 20, p. 86-91, 2014. https://doi.org/10.1016/i.esd.2014.01.006

VAN DE KAA, G. et al. How to Weigh Values in Value Sensitive Design: A Best Worst Method Approach for the Case of Smart Metering. Science and Engineering Ethics, v. 26, n. 1, p. 475-494, 2020. https://doi.org/10.1007/s11948-019-00105-3

VASSILEVA, I.; CAMPILLO, J. Consumers' perspective on full-scale adoption of smart meters: A case study in Västerås, Sweden. Resources, v. 5, n. 1, p. 3, 2016.

https://doi.org/10.3390/resources5010003

VASSILEVA, I.; CAMPILLO, J.; SCHWEDE, S. Technology assessment of the two most relevant aspects for improving urban energy efficiency identified in six mid-sized European cities from case studies in Sweden. Applied Energy, v. 194, p. 808-818, 2017.

https://doi.org/10.1016/j.apenergy.2016.07.097

WANG, S. et al. A randomized response model for privacy preserving smart metering. IEEE Transactions on Smart Grid, v. 3, n. 3, p. 1317-1324, 2012.

https://doi.org/10.1109/TSG.2012.2192487

WARKENTIN, M.; GOEL, S.; MENARD, P. Shared Benefits and Information Privacy: What Determines Smart Meter Technology Adoption? Journal of the Association for

Information Systems, v. 18, n. 11, p. 758-786, 2017. https://doi.org/10.17705/1jais.00474

WARREN, P. The potential of smart technologies and micro-generation in UK SMEs.

Energies, v. 10, n. 7, p. 1050, 2017. https://doi.org/10.3390/en10071050

WATSON, S. Consuming water smartly: the significance of sociocultural differences to water- 
saving initiatives. Local Environment, v. 22, n. 10, p. 1237-1251, 2017. https://doi.org/10.1080/13549839.2017.1334143

WEBER, M.; ŽARKO, I. P. A regulatory view on smart city services. Sensors, v. 19, n. 2, p. 415, 2019. https://doi.org/10.3390/s19020415

WEMYSS, D. et al. Examining community-level collaborative vs. competitive approaches to enhance household electricity-saving behavior. Energy Efficiency, v. 11, p. 2057-2075, 2018. https://doi.org/10.1007/s12053-018-9691-z

WILSON, C.; HARGREAVES, T.; HAUXWELL-BALDWIN, R. Benefits and risks of smart home technologies. Energy Policy, v. 103, p. 72-83, 2017.

https://doi.org/10.1016/i.enpol.2016.12.047

WOLISZ, H. et al. Feasibility and potential of thermal demand side management in residential buildings considering different developments in the German energy market.

Energy Conversion and Management, v. 107, p. 86-95, 2016.

https://doi.org/10.1016/j.enconman.2015.06.059

WUNDERLICH, P.; VEIT, D. J.; SARKER, S. Adoption of sustainable technologies: a mixedmethods study of German households. MIS Quarterly, v. 43, n. 2, p. 673-691, 2019.

https://doi.org/10.25300/MISQ/2019/12112

YANG, B. et al. Smart metering and systems for low-energy households: challenges, issues and benefits. Advances in Building Energy Research, v. 13, n. 1, p. 80-100, 2019. https://doi.org/10.1080/17512549.2017.1354782

ZHOU, S.; BROWN, M. A. Smart meter deployment in Europe: A comparative case study on the impacts of national policy schemes. Journal of Cleaner Production, v. 144, p. 22-32, 2017. https://doi.org/10.1016/j.jclepro.2016.12.031

ZHOU, S.; MATISOFF, D. C. Advanced Metering Infrastructure Deployment in the United States: The Impact of Polycentric Governance and Contextual Changes. Review of Policy Research, v. 33, n. 6, p. 646-665, 2016. https://doi.org/10.1111/ropr.12203

ZHOU, S.; NOONAN, D. S. Justice implications of clean energy policies and programs in the United States: A theoretical and empirical exploration. Sustainability, v. 11, n. 3, p. 807, 2019. https://doi.org/10.3390/su11030807

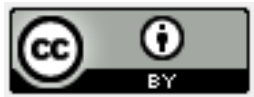

Artigo recebido em: 27/11/2020 e aceito para publicação em: 04/03/2021 DOI:http://dx.doi.org/10.14488/1676-1901.v21i1.4179 